\title{
A quantitative analysis of the role of experience in the regulation of sexual behavior in male rats
}

\author{
PABLO E. CRUZ-CASALLAS \\ University of the Llanos, Meta, Colombia \\ LUCIANO F. FELICIO \\ University of São Paulo, São Paulo, Brazil \\ and \\ ANTONIA G. NASELLO \\ Medical School of Santa Casa, São Paulo, Brazil
}

\begin{abstract}
The effects of repeated sexual behavior tests in male rats were assessed. Training (seven times, 5 days apart) revealed significant decreases in latencies to the first mount, first intromission, ejaculation, postejaculatory mount, and postejaculatory intromission, the total number of mounts, and mount frequency. The number of intromissions before ejaculation did not show differences among the seven observations. In addition, the percentage of animals that ejaculated did not change. Indices of copulatory efficiency and sexual activity were influenced by training. Both increased, but different numbers of sessions were required. Variability decreased among sessions, which increased homogeneity. Our results indicate that training (i.e., the number of test sessions) affects male sexual behavior and, therefore, should be taken into consideration when analyzing facilitatory or inhibitory influences.
\end{abstract}

Although the literature on rat male sexual behavior is abundant (for a review, see Bitran \& Hull, 1988; Meisel \& Sachs, 1994), the experimental designs used in the available reports are not always consistent, particularly with respect to whether or not the animals had received previous training. The number of times animals are exposed to receptive females before being tested experimentally also varies in the literature (Claro, Segovia, Guilamón, \& Del Abril, 1995; Liu, Salamone, \& Sachs, 1997; Nunez \& Tan, 1984; Saldivar-Gonzalez, FernandezGuasti, \& Etgen, 1993; Silva, Oliveira, Felicio, Nasello, $\&$ Bernardi, 1998). Moreover, the quantitative effects of previous sexual experience on parameters of sexual behavior, although considered in various reports, have not been the focus of detailed studies (Agmo \& Picker, 1990; Doherty, Bartke, \& Smith, 1985; Holloway \& Domjan, 1993a, 1993b; Nyby, Whitney, Schmitz, \& Dizinno, 1978). Contradictory data on the effects of hormones and neurotransmitters on sexual behavior may be due to the influence of experience on the regulation of this behavior (Doherty et al., 1985; Drago, Pellegrini-Quarantotti, Scapagnini, \& Gessa, 1981; Nasello, Vanzeler, Madureira,

We thank Fundação de Amparo a Pesquisa do Estado de São Paulo (FAPESP; Grant 96/4193-0) and Conselho Nacional de Desenvolvimento Científico e Tecnológico (CNPq; Grants 520234/96 and 520656/96) for financial support. P.E.C.-C. was supported by a fellowship from CAPES. Correspondence concerning this article should be addressed to L. F. Felicio, Departamento de Patologia, Faculdade de Medicina Veterinária, Universidade de São Paulo, Orlando M. Paiva 87, "Cidade Universitária” 05508-900, Sào Paulo, Brazil (e-mail: Ifelicio@usp.br).
\& Felicio, 1997). However, the effects of classical conditioning on male rats' sexual behavior are already known (Cruz-Casallas, Nasello, Hucke, \& Felicio, 1999; Zamble, Hadag, Mitchell, \& Cutmore, 1985; Zamble, Mitchell, \& Findlay, 1986). Some of the effects of experience on male sexual behavior have been studied previously (Beach, 1942; Larsson, 1956; Whalen, Gorzalka, \& DeBold, 1975). The purpose of this study was to analyze the effects of training (i.e., repeated sexual behavior tests in the same experimental conditions) on behavioral parameters of sexual behavior in rats, with specific emphasis on a quantitative evaluation of the reduction in latency induced by previous training and on the effect of training on other parameters of male rat sexual behavior. In addition, the implications of these parameters for research on sexual behavior are discussed - that is, the possibility that previous sexual experience masks facilitatory influences or discloses disruptive influences on this behavior. To further our knowledge concerning the influence of this physiological variable, this experiment was designed to assess the patterns of variation of the various parameters of male rat sexual behavior during the first seven sessions, held at 5-day intervals.

\section{METHOD}

Twenty naive male Wistar rats, weighing $250-300 \mathrm{~g}$, about 3-4 months old, were used. The animals were housed in polypropylene cages $(32 \times 40 \times 15 \mathrm{~cm}), 4$ per cage, under controlled temperature $\left(22^{\circ}-23^{\circ} \mathrm{C}\right)$, and were submitted to reversed lighting conditions (lights off from 10:00 to 22:00 h). All the behavioral tests were con- 
Table 1

Influence of Training on Sexual Behavior of Inexperienced Male Rats During the First Seven Sessions

\begin{tabular}{lccccccc}
\hline & \multicolumn{5}{c}{ Test } \\
\cline { 2 - 7 } \multicolumn{1}{c}{ Behavior } & 1 & 2 & 3 & 4 & 5 & 6 \\
\hline Ejaculation frequency & $90 \%$ & $95 \%$ & $100 \%$ & $100 \%$ & $10 \%^{*}$ & $100 \%$ & $100^{*}$ \\
Mount latency & $94 \pm 29$ & $17 \pm 4$ & $15 \pm 4^{*}$ & $8 \pm 2^{*}$ & $5 \pm 1^{*}$ & $5 \pm 1^{*}$ & $4 \pm 1^{*}$ \\
Intromission latency & $104 \pm 29$ & $48 \pm 16$ & $16 \pm 4^{*}$ & $9 \pm 2^{*}$ & $6 \pm 1^{*}$ & $7 \pm 1^{*}$ & $5 \pm 1^{*}$ \\
Ejaculation latency & $378 \pm 42$ & $345 \pm 34$ & $275 \pm 37$ & $241 \pm 34^{*}$ & $204 \pm 22^{*}$ & $233 \pm 24^{*}$ & $209 \pm 24^{*}$ \\
Postejaculatory mount latency & $346 \pm 16$ & $322 \pm 13$ & $316 \pm 11$ & $323 \pm 10$ & $295 \pm 10^{*}$ & $307 \pm 12$ & $295 \pm 13^{*}$ \\
Postejaculatory intromission latency & $359 \pm 16$ & $332 \pm 12$ & $324 \pm 11$ & $326 \pm 10$ & $297 \pm 10^{*}$ & $309 \pm 12^{*}$ & $298 \pm 13^{*}$ \\
\hline
\end{tabular}

${ }^{*} p<.05$, as compared with Day 1 (Kruskal-Wallis/Mann-Witney $U$ test). Data are means $\pm S E M(n=20)$. All latencies are in seconds.

ducted between 13:00 and 18:00 $\mathrm{h}$, during the dark phase of the light cycle. Food and water were available ad lib.

\section{Experiment and Behavioral Testing}

Seven sexual behavioral tests were held at 5-day intervals, using an observation cage $(56 \times 32 \times 32 \mathrm{~cm})$ with pine shavings on the floor, illuminated by $25-\mathrm{W}$ red lamps. Males were allowed to acclimate to the behavior arena for at least $5 \mathrm{~min}$. A receptive female, treated as has been described elsewhere (Felicio \& Nasello, 1989; Felicio, Palermo-Neto, \& Nasello, 1989), was then introduced. The test lasted until the first intromission after ejaculation or, if no ejaculation occurred, $15 \mathrm{~min}$ after the introduction of the female into the observation cage. The following parameters were recorded: (1) mount latency, (2) intromission latency, (3) number of incomplete mounts (mounts without intromission) before ejaculation, (4) number of intromissions (before ejaculation, including the ejaculatory one), (5) ejaculation latency, (6) postejaculatory mount latency, and (7) postejaculatory intromission latency. In addition, four derived measures of the intensity of sexual behavior were calculated: (1) intromission frequency (number of intromissions $\cdot$ time $^{-1}$ ), (2) mount frequency (number of intromissions plus number of mounts divided by the time from first mount to ejaculation), (3) hit rate (number of intromissions divided by number of intromissions plus number of mounts), and finally (4) an overall measure of sexual activity, the sexual activity index (SAI), as proposed by Agmo, Paredes, and Fernández (1987). The SAI was calculated in the following way: The inverse values of the mount, intromission, and ejaculation latencies (ML, IL, and EL, respectively) were multiplied by the test period in minutes (i.e., 15) and then transformed into logarithms; the number of mounts plus intromissions was transformed into a square root; the presence of ejaculation was assigned a value of 4 , and the absence of ejaculation a value of 0 . The sum of the transformed numbers constitutes the SAI-that is, $\mathrm{SAI}=\log \{(1 / \mathrm{ML}) \times 15\}+\log$ $\{(1 / \mathrm{IL}) \times 15\}+\log \{(1 / \mathrm{EL}) \times 15\}+\sqrt{ } \mathrm{M}+4$ (if ejaculation occurs) or 0 (if ejaculation does not occur). All the parameters were calcu- lated by considering only animals that ejaculated, except for the SAI. In addition, the percentage of animals showing ejaculation was recorded.

\section{Statistical Analysis}

Since Bartlet's test did not show the existence of homogeneity among the data, behavioral measures were compared with a KruskalWallis nonparametric analysis of variance (ANOVA), followed by the Mann-Witney $U$ test. In all cases, differences were considered significant if $p<.05$.

\section{RESULTS}

Seven sessions of training experience significantly influenced various parameters of male sexual behavior. A Kruskal-Wallis ANOVA revealed significant decreases in latencies to first mount $(\mathrm{KW}=84.170, p<.0001$ Table 1), first intromission ( $\mathrm{KW}=84.197, p<.0001$; Table 1), ejaculation $(\mathrm{KW}=35.148, p<.0001$; Table 1$)$, postejaculatory mount $(\mathrm{KW}=13.896, p<.05$; Table 1$)$, postejaculatory intromission $(\mathrm{KW}=17.533, p<.01$; Table 1), total number of mounts $(\mathrm{KW}=12.623, p<.05$; Table 2), and mount frequency (KW $=47.237, p<.0001$; Table 2). Number of intromissions before ejaculation did not show significant differences among the seven observations (Table 2). The percentage of animals that ejaculated did not change significantly across sessions ( $\mathrm{Ta}$ ble 1). Post hoc comparisons revealed significant decreases in mount and intromission latencies on Tests 3, 4, 5, 6, and 7 , as compared with the first testing day (Day 0 ). A significant decrease in ejaculation latency was observed

Table 2

Influence of Training on Sexual Behavior of Inexperienced Male Rats During the First Seven Sessions

\begin{tabular}{lccccccc}
\hline & \multicolumn{7}{c}{ Test } \\
\cline { 2 - 8 } \multicolumn{1}{c}{ Behavior } & 1 & 2 & 3 & 4 & 5 & 6 & 7 \\
\hline Number of incomplete mounts & $4.2 \pm 0.8$ & $8.9 \pm 1.4^{*}$ & $4.7 \pm 1.0$ & $3.6 \pm 1.0$ & $3.5 \pm 0.8$ & $5.4 \pm 1.0$ & $4.8 \pm 1.4$ \\
Number of intromissions & $8.6 \pm 0.5$ & $9.3 \pm 0.6$ & $10.6 \pm 0.6$ & $9.9 \pm 0.7$ & $10.4 \pm 0.6$ & $10.2 \pm 0.6$ & $9.1 \pm 0.5$ \\
Total number of mounts (incomplete & & & & & & & \\
$\quad$ mounts + intromissions) & $12.8 \pm 1.0$ & $18.2 \pm 1.2$ & $15.3 \pm 1.2$ & $13.5 \pm 1.4$ & $13.9 \pm 1.2$ & $15.1 \pm 1.2$ & $13.9 \pm 1.5$ \\
Intromissions per minute & $1.5 \pm 0.2$ & $1.7 \pm 0.2$ & $2.9 \pm 0.3^{*}$ & $2.9 \pm 0.4^{*}$ & $3.7 \pm 0.4^{*}$ & $3.0 \pm 0.3^{*}$ & $3.6 \pm 0.5^{*}$ \\
Mounts per minute & $2.0 \pm 0.2$ & $2.9 \pm 0.2$ & $3.7 \pm 0.3^{*}$ & $3.8 \pm 0.4^{*}$ & $4.6 \pm 0.4^{*}$ & $4.2 \pm 0.3^{*} 4.8 \pm 0.5^{*}$ \\
\hline${ }^{*} p<.05$, as compared with Day 1 (Kruskal-Wallis/Mann-Witney $U$ test). Data are means $\pm S E M(n=20)$.
\end{tabular}



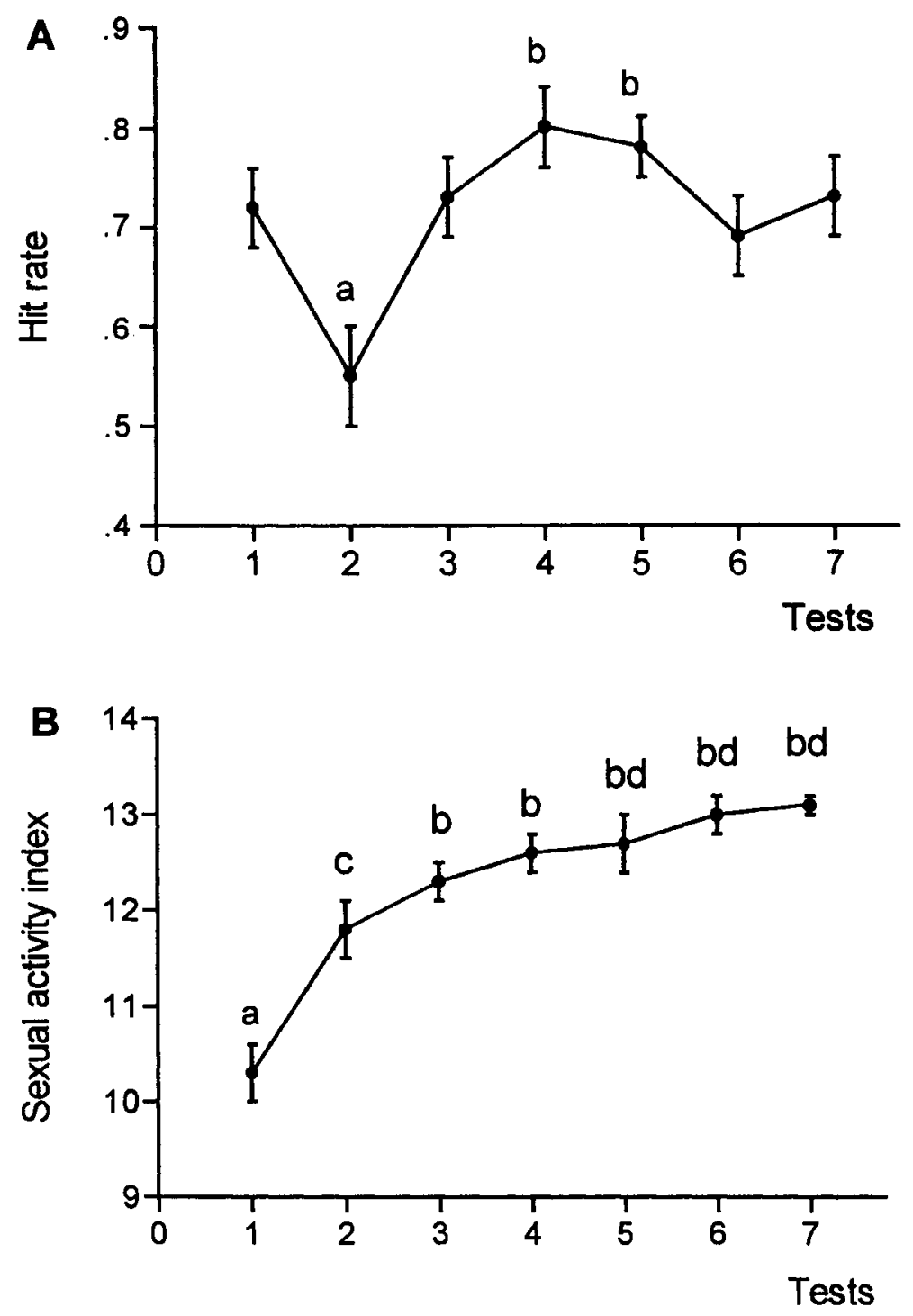

Figure 1. Effect of experience on hit rate (A) and sexual activity index (B) of rats. Data are means $\pm S E M(n=20)$. The sexual activity index was calculated as follows: The inverse values of the mount (ML), intromission (IL), and ejaculation (EL) latencies were multiplied by 15 and then transformed into natural logarithms; the number of mounts plus intromissions was transformed into a square root; the presence of ejaculation was assigned a value of 4 , and the absence of ejaculation a value of 0 . The sum of the transformed numbers constitutes the sexual activity index--that is, SAI $=\log \{(1 / M L) \times 15\}+\log \{(1 / I L)$ $\times 15\}+\log \{(1 / E L) \times 15\}+\sqrt{ } M+4$ (if ejaculation occurs) or 0 (if ejaculation does not occur). A Kruskal-Wallis analysis of variance, followed by MannWhitney's $U$ test, showed differences among $a$ and $b, c$ and $d$.

from Test 4 to Test 7 . Postejaculatory mount and intromission latencies decreased significantly on Tests 5 and 7 and from Test 5 to Test 7 , respectively (Table 1). Indices of hit rate $(\mathrm{KW}=17.708, p<.01$; Figure 1$)$ and of sexual activity $(\mathrm{KW}=61.819, p<.05)$ were both influenced by training. Hit rate was significantly lower on Test 2 than on Tests 4 and 5, but no other significant differences among days were observed for this parameter. Sexual activity increased significantly from Test 3 to
Test 7, as compared with the first training sessions. From Test 5 to Test 7, this index was significantly higher than on Test 2.

\section{DISCUSSION}

The present results show a clear experience-induced quantitative improvement in sexual performance that is positively related to number of sessions. However, the 
parameters were modified in different ways by training. Motivational parameters - that is, latencies for mount and intromission - were more sensitive to experienceinduced changes than were the consumatory parameters, such as those involving ejaculation (i.e., number of intromissions, latency for ejaculation, and postejaculatory mount and intromission latencies). All the latencies significantly decreased, but the decrease in ejaculatory latency was less intense. This shows an intrinsic learning that depends on the number of sessions, when they are repeated in the same experimental conditions for several weeks. The influence of classical (Pavlovian) conditioning in rat sexual behavior and its importance for human sexual problems have been studied already (Cutmore \& Zamble, 1988; Larsson, 1956; Zamble et al., 1985; Zamble et al., 1986). Sexual approach conditioning and other mechanisms of learning have also been described, but not for mammalian species (Claro et al., 1995; Domjan, Akins, \& Vandergriff, 1992; Holloway \& Domjan, 1993a, 1993b). The copulatory efficiency did not change significantly, except for the second trial. SAI, which involves all of the above parameters, increased significantly after the second session, showing an improvement in sexual behavior as a whole (Agmo et al., 1987). The results suggest that experience influences certain parameters and does not interfere with others. Variability decreased in the course of the sessions, increasing group homogeneity. Changes occurred in the same direction when both absolute and percentage data are considered. On the basis of the present data, we conclude that in male sexual behavior studies, it is necessary to control for experience and to report whether the subject has been submitted to one or more previous experiences. In the latter case, the number of sessions should be mentioned.

Contradictory reports on short-term hyperprolactinemia facilitatory effects on male sexual behavior illustrate this point, and the contradictory results may be due to the influence of experience on the regulation of this behavior. Although the effect of prolactin on male sexual behavior changes from facilitatory (acute effects) to inhibitory (long-term effects), the prolactin-induced facilitation is disclosed in naive rats and concealed, or nonexistent, in trained animals (Cruz-Casallas et al., 1999; Doherty et al., 1985; Drago et al., 1981). To evaluate influences of sensory or chemical stimuli on this behavior, experimental and control animals must have been exposed to the same number of training sessions. To discuss facilitatory or inhibitory influences on the various parameters of male sexual behavior, it is necessary to determine whether changes occur in parameters sensitive to training, do not so occur, or both (Claro et al., 1995). It is important to consider that sexual experience may mask facilitatory influences on this behavior (Nasello et al., 1997). On the other hand, training experience, by decreasing latencies and variability, may reveal disruptive influences (Holloway \& Domjan, 1993a). Since a decrease in intragroup variability is obtained with training, this may be useful for certain experimental designs and may eventually help to disclose influences of different inhibitory stimuli. Questions related to modulatory effects of experience on male sexual behavior, such as the importance of interaction between number of tests and the intervals between the tests, as well as the brain mechanisms involved in this phenomenon, are still to be addressed. Future experiments will need to be designed to approach those issues.

\section{REFERENCES}

Agmo, A., \& Picker, Z. (1990). Catecholamines and the initiation of sexual behavior in male rats without sexual experience. Pharmacology, Biochemistry \& Behavior, 35, 327-334.

Agmo, A., Paredes, R., \& Fernández, H. (1987). Differential effects of GABA transaminase inhibitors on sexual behavior, locomotor activity, and motor execution in the male rat. Pharmacology, Biochemistry \& Behavior, 28, 47-52.

BEACH, F. A. (1942). Analysis of factors involved in the arousal, maintenance and manifestation of sexual excitement in male animals. Psychosomatic Medicine, 4, 173-198.

Bitran, D., \& Hull, E. M. (1988). Pharmacological analysis of male rat sexual behavior. Neuroscience \& Biobehavioral Reviews, 20 , 323-331.

Claro, F., Segovia, S., Guilamón, A., \& Del Abril, A. (1995). Lesions in the medial posterior region of the BST impair sexual behavior in sexually experienced and inexperienced male rats. Brain Research Bulletin, 36, 1-10.

Cruz-Casallas, P. E., Nasello, A. G., Hucke, E. E. T, S., \& Felicio, L. F. (1999). Central prolactin dual modulation of male sexual behavior in rats: Relationship with in vivo striatal dopaminergic activity. Psychoneuroendocrinology, 24, 681-693.

CUTMORE, T. R., \& ZAMBLE, E. A. (1988). Pavlovian procedure for improving sexual performance of noncopulating male rats. Archives of Sexual Behavior, 17, 371-380.

DoherTy, P. C., BARTKE, A., \& SMITH, M. S. (1985). Hyperprolactinemia and male sexual behavior: Effects of steroid replacement with estrogen plus dihydrotestosterone. Physiology \& Behavior, 35, 99-104.

Dom jan, M., Akins, C., \& Vandergriff. D. H. (1992). Increased responding to female stimuli as a result of sexual experience: Tests of mechanisms of learning. Quarterly Journal of Experimental Psychology, 45B, 139-157.

Drago, F., Pellegrini-Quarantotti, B., Scapagnini, U., \& Gessa, G. L. (1981). Short-term endogenous hyperprolactinemia and sexual behavior of male rats. Physiology \& Behavior, 26, 277-279.

FELICIO, L. F., \& NASELlo, A. G. (1989). Effects of acute bromopride treatment on rat prolactin levels and sexual behavior. Brazilian Journal of Medical \& Biological Research, 22, 1011-1014.

Felicio, L. F., Palermo-Neto, J., \& Nasello, A. G. (1989). Perinatal bromopride treatment: Effects on sexual behavior of male and female rats. Behavioral \& Neural Biology, 52, 145-151

Holloway, K. S., \& DOM JAN, M. (1993a). Sexual approach conditioning: Tests of unconditioning stimulus devaluation using hormone manipulations. Journal of Experimental Psychology: Animal Behavior Processes, 19, 47-55.

Holloway, K. S., \& Dom Jan, M. (1993b). Sexual approach conditioning: Unconditioned stimulus factors. Journal of Experimental Psychology: Animal Behavior Processes, 19, 38-46.

LARSSON, K. (1956). Conditioning and sexual behavior in the male albino rat. Stockholm: Almqvist \& Wiksell.

LiU, Y.-C., SALAmone, J. D., \& SaChs, B. D. (1997). Lesions in medial preoptic area and bed nucleus of stria terminalis: Differential effects on copulatory behavior and noncontact erection in male rats. Journal of Neuroscience, 1, 5245-5253.

MEISEL, R. L., \& SACHS, B. D. (1994). The physiology of male sexual 
behavior. In E. Knobil \& J. D. Neill (Eds.), The physiology of reproduction (2nd ed., Vol. 2; pp. 3-105). New York: Raven.

Nasello, A. G., Vanzeler, M. L. A., Madureira, E. H., \& Felicio, L. F. (1997). Effects of acute and long-term domperidone treatment on prolactin and gonadal hormone levels and sexual behavior of male and female rats. Pharmacology, Biochemistry \& Behavior, 58, 1089-1094.

Nunez, A. A., \& TAN, D. T. (1984). Courtship ultrasonic vocalizations in male Swiss-Webster mice: Effects of hormones and sexual experience. Physiology \& Behavior, 32, 717-721.

Nyby, J., Whitney, G., Schmitz, S., \& Dizinno, G. (1978). Postpubertal experience establishes signal value of mammalian sex odor. Behavioral Biology, 22, 545-552.

Saldivar-Gonzalez, A., Fernandez-Guasti, A., \& Etgen, A. M. (1993). Male rat sexual behavior induces changes in $\left[{ }^{3} \mathrm{H}\right]$ flunitrazepam binding. Brain Research, 611, 326-329.

Silva, M. R. P., Oliveira, C. A., Felicio L. F., Nasello, A. G., \& BERNARDI, M. M. (1998). Perinatal treatment with picrotoxin induces sexual behavioral and neuroendocrine changes in male rats. Pharmacology, Biochemistry \& Behavior, 60, 203-208.

Whalen, R. E., Gorzalka, B. B., \& DeBold, J. F. (1975). Methodologic considerations in the study of animal sexual behavior. In M. Sandler \& G. L. Gessa (Eds.), Sexual behavior: Pharmacology \& biochemistry (pp. 33-440) New York: Raven.

Zamble, E., HadaG, G. M., Mitchell, J. B., \& CutmoRe, T. R. (1985). Pavlovian conditioning of sexual arousal: First- and second-order effects. Journal of Experimental Psychology: Animal Behavior Processes, 11, 598-610.

Zamble, E., Mitchell, J. B., \& Findlay, H. (1986). Pavlovian conditioning of sexual arousal: Parametric and background manipulations. Journal of Experimental Psychology: Animal Behavior Processes, $12,403-411$.

(Manuscript received October 21, 1999; revision accepted for publication March 1,2000.) 